\title{
Erratum to: The challenge of acute rejection in intestinal transplantation
}

\author{
E. Y. Yoshitoshi $\cdot$ A. Yoshizawa $\cdot$ E. Ogawa $\cdot$ M. Kaneshiro $\cdot$ N. Takada \\ S. Okamoto $\cdot$ Y. Fujimoto $\cdot$ S. Sakamoto $\cdot$ S. Masuda $\cdot$ M. Matsuura $\cdot$ \\ H. Nakase $\cdot$ T. Chiba $\cdot$ T. Tsuruyama $\cdot$ H. Haga $\cdot$ S. Uemoto
}

Published online: 17 February 2013

(C) Springer-Verlag Berlin Heidelberg 2013

Erratum to: Pediatr Surg Int (2012) 28:855-859

DOI 10.1007/s00383-012-3110-x

The co-author H. Nakase was incorrectly listed as Y. Nakase; this error is corrected here.

The online version of the original article can be found under doi:10.1007/s00383-012-3110-x.

E. Y. Yoshitoshi $(\bowtie) \cdot$ A. Yoshizawa $\cdot$ E. Ogawa

M. Kaneshiro · N. Takada - S. Okamoto · Y. Fujimoto ·

S. Uemoto

Department of Hepatobiliary, Pancreas, Transplantation and Pediatric Surgery, Kyoto University Hospital,

Graduate School of Medicine, 54 Kawara-cho,

Shogoin, Sakyo-ku, Kyoto city 606-8507, Japan

e-mail: elenayy@yahoo.com

\section{S. Sakamoto}

Department of Transplant Surgery, National Center for Child

Health and Development, 2-10-1 Okura, Setagaya-ku,

Tokyo 157-8535, Japan

\section{S. Masuda}

Department of Pharmacology, Kyoto University Hospital,

Graduate School of Medicine, 54 Kawara-cho,

Shogoin, Sakyo-ku, Kyoto-shi 606-8507, Japan
M. Matsuura - H. Nakase - T. Chiba

Department of Gastroenterology, Kyoto University Hospital,

Graduate School of Medicine, 54 Kawara-cho,

Shogoin, Sakyo-ku, Kyoto-shi 606-8507, Japan

T. Tsuruyama $\cdot$ H. Haga

Department of Pathology, Kyoto University Hospital,

Graduate School of Medicine, 54 Kawara-cho,

Shogoin, Sakyo-ku, Kyoto-shi 606-8507, Japan 